\title{
A numerical investigation of ductile fracture initiation in a high-strength low-alloy steel
}

\author{
R NARASIMHAN and S V KAMAT* \\ Department of Mechanical Engineering, Indian Institute of Science, Bangalore 560012, \\ India \\ * Defence Metallurgical Research Laboratory, Hyderabad 500258, India \\ MS received 20 October 1993; revised 17 March 1994
}

\begin{abstract}
In this work, static and drop-weight impact experiments, which have been conducted using three-point bend fracture specimens of a high-strength low-alloy steel, are analysed by performing finite-element simulations. The Gurson constitutive model that accounts for the ductile failure mechanisms of microvoid nucleation, growth and coalescence is employed within the framework of a finite deformation plasticity theory. Two populations of second-phase particles are considered, including large inclusions which initiate voids at an early stage and small particles which require large strains to nucleate voids. The most important objective of the work is to assess quantitatively the effects of material inertia, strain rate sensitivity and local adiabatic temperature rise (due to conversion of plastic work into heat) on dynamic ductile crack initiation. This is accomplished by comparing the evolution histories of void volume fraction near the notch tip in the static analysis with the dynamic analyses. The results indicate that increased strain hardening caused by strain rate sensitivity, which becomes important under dynamic loading, plays a benign role in considerably slowing down the void growth rate near the notch tip. This is partially opposed by thermal softening caused by adiabatic heating near the notch tip.
\end{abstract}

Keywords. Ductile fracture; finite elements; impact loading.

\section{Introduction}

Ductile fracture is caused mainly by the micromechanical processes of void nucleation, growth and coalescence. Voids nucleate due to brittle cracking or interfacial decohesion of inclusions and then grow by plastic deformation of the surrounding material. It has been observed (see, for example, Cox and Low 1974; Hahn and Rosenfield 1975) that in structural materials such as steels and aluminium alloys void initiation can involve two distinct populations of inclusions. The voids nucleate first at large inclusions, and after growing to some size, they coalesce, or link up with a nearby crack tip via a void sheet consisting of voids nucleated from smaller particles.

The initiation of fracture under dynamic loading, such as that produced by impact or explosive detonation, is relevant to several important engineering structures. In such situations, the loading rates (measured in terms of the stress intensity rate $\dot{K}$ ) could be much higher (more than $10^{4}$ times) than in a conventional quasistatic fracture test. For many metallic materials, it has been observed from experiments (see, for example, Priest 1976) that the dynamic fracture toughness $K_{I d}$ depends strongly on $\dot{K}$. It is believed that several factors such as material inertia, strain rate sensitivity, and adiabatic heating near the crack tip can influence the functional dependence of $K_{I d}$ on $\dot{K}$. However, a detailed study that clearly delineates the individual role played by the above factors and also their combined effect on the ductile fracture process under dynamic loading has not been carried out so far in the literature. Such a 
fundamental investigation is imperative in order to be able to develop materials which will be better suited to resist dynamic failure. It is of interest from a design standpoint for such applications as are envisaged here that the fracture toughness of the material should increase with loading rate $\dot{K}$.

Notwithstanding what has been mentioned above, it is important to recognize that several studies have been undertaken to model the micromechanics of ductile fracture. In some early investigations, Rice and Johnson (1970) and McMeeking (1977) examined the interaction between a void situated near a crack tip and the intense deformation fields generated due to the blunting of the crack tip. In recent years, many investigators have used damage accumulation models like the Gurson (1977) model to study ductile failure under various situations. Tvergaard and Needleman (1984) analyzed cup and cone fracture in a round tensile bar using the Gurson model to represent the material behaviour. Needleman and Tvergaard (1987) studied fracture initiation in a ductile material involving two sets of particles of different sizes. A similar analysis was undertaken by Needleman and Tvergaard (1991) to simulate dynamic ductile crack propagation.

Narasimhan (1992) carried out finite-element analyses of static and impact (dynamic) fracture experiments, involving pre-notched 4340 steel specimens, which had been conducted by Zehnder et al (1990). In his work, attention was focused on the formation of a discrete void around a simulated inclusion ahead of the notch tip, its growth and eventual link up with the notch tip. It was observed by Narasimhan (1992) that there is a close correlation between the time variation of the $J$ integral and the accumulation of void volume fraction near the notch tip (under dynamic loading). Further, it was found that, due to the influence of material inertia, a larger value of $J$ is necessary to achieve the same level of ductile damage near the notch tip under dynamic loading compared to the static case.

In the present work, an investigation similar to that conducted by Narasimhan (1992) is performed to assess quantitatively the effects of material inertia, strain rate sensitivity and local adiabatic temperature rise (due to conversion of plastic work into heat) on dynamic ductile crack initiation. To this end, finite-element analyses are carried out of the static and drop-weight impact experiments conducted at the Defence Metallurgical Research Laboratory (DMRL), Hyderabad, using three-point bend fracture specimens. The material employed in these experiments is a highstrength low-alloy steel (called SPADE) developed at DMRL. The static fracture experiments and uniaxial tension tests were carried out on an Instron Servo-hydraulic testing machine, while the impact tests were performed using a Dynatup drop-weight tower with an initial impact speed of $6 \mathrm{~m} / \mathrm{s}$. The information gathered from the experiments regarding the uniaxial behaviour of the material, the time variation of load (in the impact test), etc. are employed in the finite-element analyses conducted in this work.

As in the previous work by Narasimhan (1992), void initiation at two populations of particles is considered. The initiation of voids at small (submicron size), uniformly distributed particles is assumed to be based on a strain-controlled mechanism. On the other hand, a stress-controlled void nucleation law is used within the framework of the Gurson constitutive model to simulate the formation of discrete voids at large inclusions surrounding the notch tip (see $\$ 2$ for details). The impact fracture experiment is modelled thrice by dynamic finite-element analyses (see $\S 3$ ). The 
objective of these three analyses is to study the individual roles of thermal softening (caused by adiabatic heating) and strain rate sensitivity, in addition to material inertia, and also their combined effect on the ductile fracture process. The dynamic finiteelement analyses are supplemented by an elementary analysis using the mass-spring model of Williams (1987) to compute the time variation of global parameters like specimen energies and the $J$ integral.

\section{Description of the constitutive model}

In this work, the constitutive equations proposed by Gurson (1977) are employed which are based on a continuum elastic-plastic model that accounts for microvoid nucleation and growth. This model was subsequently modified by Tvergaard and Needleman (1984), since in its original form the complete loss of material stresscarrying capacity due to microvoid coalescence was not predicted at a realistic level of void volume fraction. The modified Gurson yield condition which depends on the macroscopic (aggregate) Cauchy stress $\sigma_{i j}$, the microscopic (matrix) tensile flow stress $\sigma_{m}$, and the current void volume fraction $f$ is given by

$$
\Phi\left(\sigma_{i j}, \sigma_{m}, f\right)=\frac{\sigma_{e}^{2}}{\sigma_{m}^{2}}+2 f^{*} q_{1} \cosh \left(\frac{3 \sigma_{H}}{2 \sigma_{m}}\right)-\left(1+\left(q_{1} f^{*}\right)^{2}\right)=0 .
$$

(Standard Cartesian tensor notation is employed in this paper with repeated indices implying summation.) It should be noted that the above yield condition depends both on the deviatoric component $S_{i j}$ of the Cauchy stress tensor through the macroscopic equivalent stress $\sigma_{e}=\left(3 / 2 S_{i j} S_{i j}\right)^{1 / 2}$ and also on the hydrostatic stress $\sigma_{H}=\sigma_{k k} / 3$. This model thus exhibits a dilatational plastic behaviour which is an outcome of the presence of microvoids inside the matrix. Tvergaard (1981) introduced the parameter $q_{1}$ in (1), with a value of 1.5 , to obtain better agreement between predictions of this model and numerical studies of periodic array of voids.

The modelling of final material failure by microvoid coalescence is accomplished through the function $f^{*}(f)$ in (1) which is defined by

$$
f^{*}= \begin{cases}f & f \leqslant f_{c} \\ f_{c}+K\left(f-f_{c}\right) & f>f_{c}\end{cases}
$$

where $K=\left(1 / q_{1}-f_{c}\right) /\left(f_{F}-f_{c}\right)$. Here, $f_{c}$ is the value of the void volume fraction at which void coalescence first occurs and $f_{F}$ is its value at final failure. From (2) it is clear that as $f \rightarrow f_{F}, f^{*} \rightarrow f_{u}^{*}=1 / q_{1}$. Further, it can be observed from the yield condition (1) that the material loses its stress-carrying capacity when $f^{*} \rightarrow f_{u}^{*}=1 / q_{1}$ : Thus, an essential feature of the model is that a failure criterion is directly built into the constitutive equations. Experimental studies (Brown and Embury 1973; Cox and Low 1974; Goods and Brown 1979) indicate that the ligament connecting two neighbouring voids fails by shear band formation or by simple necking when the size of the voids has grown to the order of magnitude of their spacing. An estimate of $f_{c}$ obtained by Brown and Embury (1973) from a simple model is $0 \cdot 15$. Also, a numerical investigation by Andersson (1977) suggests that $f_{F} \approx 0.25$.

The total deformation rate $D_{i j}$ (which is the symmetric part of the spatial gradient 
of velocity) is taken to be the sum of an elastic part, a plastic part and a part due to thermal straining. Thus,

$$
D_{i j}=D_{i j}^{e}+D_{i j}^{p}+D_{i j}^{T} \text {. }
$$

The Jaumann rate [the Jaumann rate (which is an objective rate) of the Cauchy stress tensor is defined by $\sigma_{i j}^{*}=\dot{\sigma}_{i j}+\sigma_{i k} W_{k j}-W_{i k} \sigma_{k j}$, where $W_{i j}$ is the spin rate (skew symmetric part of the spatial gradient of velocity)] of the Cauchy stress $\sigma_{i j}^{*}$ is related to $D_{i j}^{e}$ by a constant, positive definite, isotropic elasticity tensor $C_{i j k l}$ as

$$
\sigma_{i j}^{*}=C_{i j k l} D_{k l}^{e} \text {. }
$$

The flow rule is assumed to conform to macroscopic normality, so that

$$
D_{i j}^{p}=\dot{\lambda} \frac{\partial \Phi}{\partial \sigma_{i j}},
$$

where $\dot{\lambda} \geqslant 0$ is a plastic parameter.

The deformation rate due to thermal straining, $D_{i j}^{T}$, can be written as

$$
D_{i j}^{T}=\alpha \dot{T} \delta_{i j}
$$

where $\alpha$ is the thermal expansion coefficient and $\dot{T}$ is the temperature rate. The temperature rise is caused by plastic dissipation. It is assumed to be negligible under static loading, while adiabatic conditions are assumed to prevail (near the notch tip) under dynamic (drop-weight) loading, so that

$$
\dot{T}=\frac{\chi}{\rho_{o} c_{p}} \mathscr{J} \sigma_{i j} D_{i j}^{p}
$$

Here $\rho_{o}$ is the mass density in the reference configuration, $c_{p}$, the heat capacity, and $\mathscr{F}$, the Jacobian of the deformation. The parameter $\chi$ in (7) specifies the fraction of plastic work that is converted into heat. The value of $\chi$ is taken as 0.9 , which is typical for metals (Taylor and Quinney 1934).

The matrix material behaviour is represented by a thermally softening plastic solid. The matrix flow stress $\sigma_{m}$ is taken to depend on the matrix plastic strain $\varepsilon_{m}^{p}$ and the temperature $T$ in the following manner:

$$
\sigma_{m}=\sigma_{o} f\left(\varepsilon_{m}^{p}\right) g(T) \text {. }
$$

Here $\sigma_{o}$ is a reference stress, and $f\left(\varepsilon_{m}^{p}\right)$ and $g(T)$ are functions that characterize strain hardening and thermal softening respectively. They are chosen as

and

$$
f\left(\varepsilon_{m}^{p}\right)=\left(1+\varepsilon_{m}^{p} / \varepsilon_{o}\right)^{N},
$$

$$
g(T)=1-\gamma\left(T-T_{o}\right)
$$

In the above equations, $\varepsilon_{o}=\sigma_{o} / E, N$ is the strain hardening exponent, $T_{o}$, a reference temperature and $\gamma$, a thermal softening parameter.

The evolution law for $\sigma_{m}$ is derived by setting the macroscopic plastic work rate 
equal to the microscopic plastic dissipation, so that

$$
\sigma_{i j} D_{i j}^{p}=(1-f) \sigma_{m} \dot{\varepsilon}_{m}^{p} .
$$

The void volume fraction $f$ is allowed to evolve both due to growth of existing voids and nucleation of new voids, so that

$$
\dot{f}=\dot{f}_{\text {growth }}+\dot{f}_{\text {nucleation }} \text {. }
$$

The growth law, which is described by

$$
\dot{f}_{\text {growth }}=(1-f) D_{k k}^{p} \text {, }
$$

is an outcome of the plastic incompressibility of the matrix material. It should, however, be noted that the macroscopic material response does not satisfy plastic incompressibility due to the existence of voids.

The void nucleation law that is used in this work is of the form

$$
\dot{f}_{\text {nucleation }}=\mathscr{A} \dot{\varepsilon}_{m}^{p}+\mathscr{B}\left(\dot{\sigma}_{m}+c \dot{\sigma}_{H}\right),
$$

which was proposed by Needleman and Rice (1978). In this equation, $c$ is a constant less than unity, and $\mathscr{A}(\cdot)$ and $\mathscr{B}(\cdot)$ are considered as functions of $\varepsilon_{m}^{p}$ and $\sigma_{m}+c \sigma_{H}$ respectively. The choice of these functional forms is discussed below.

A plastic strain-controlled void nucleation law can be employed (see Thomason 1990) to model void nucleation at small particles (less than $1 \mu \mathrm{m}$ in size) which can be assumed to be uniformly distributed in the matrix. In this case, the function $\mathscr{B} \equiv 0$, and the function $\mathscr{A}\left(\varepsilon_{m}^{p}\right)$ is chosen as (Chu and Needleman 1980)

$$
\mathscr{A}=\frac{f_{n}}{s_{n} \sqrt{2 \pi}} \exp \left[-\frac{1}{2}\left(\frac{\varepsilon_{m}^{p}-\varepsilon_{n}}{s_{n}}\right)^{2}\right],
$$

so that void nucleation follows a normal distribution about a mean nucleation strain $\varepsilon_{n}$ with standard deviation $s_{n}$. In the above, $f_{n}$ denotes the volume fraction of void nucleating particles which initiate voids by a plastic strain-controlled mechanism.

In order to represent microvoid nucleation at large-size inclusions (greater than about $1 \mu \mathrm{m}$ ), a model based on the maximum normal stress at the particle-matrix interface should be used. This idea has been suggested by the works of Argon and coworkers (Argon and Im 1975; Argon et al 1975). The above mechanism can be represented through the nucleation law (13) by taking the function $\mathscr{A} \equiv 0$ and by choosing the function $\mathscr{B}\left(\sigma_{m}+c \sigma_{H}\right)$ to have a normal distribution about a mean nucleation stress $\sigma_{N}$, so that

for

$$
\mathscr{B}=\frac{\hat{f}_{n}}{\hat{s}_{n} \sqrt{2 \pi}} \exp \left[-\frac{1}{2}\left(\frac{\left(\sigma_{m}+c \sigma_{H}\right)-\sigma_{N}}{\hat{s}_{n}}\right)^{2}\right]
$$

$$
\left(\sigma_{m}+c \sigma_{H}\right)=\left(\sigma_{m}+c \sigma_{H}\right)_{\max } \text { and }\left(\dot{\sigma}_{m}+c \dot{\sigma}_{H}\right)>0 .
$$

Here $\hat{f}_{n}$ is the volume fraction of particles that initiate voids by the mechanism described above which is based on the interface stress, and $\hat{s}_{n}$ is a standard deviation 
about the mean nucleation stress $\sigma_{N}$. If the condition $(15 b)$ is violated then $\mathscr{B}$ is taken as zero. The value of $c$ is taken as less than unity in order to account for the partial conversion of the remote hydrostatic stress into local shearing stress around the inclusion.

In order to realistically simulate the discrete nature of void initiation at the sites of large-size particles which fail by an interface stress-controlled mechanism, the approach suggested by Needleman and Tvergaard (1987) is employed. Here, the large particles are modelled as islands (i.e. as contours which enclose the centres of the particles) of the amplitude $\hat{f}_{n}$ of the stress-controlled void nucleation function (15a). Thus, considering the large particles to be distributed in a rectangular array and to be circular-cylindrical in shape, in the spirit of the 2-D plane strain analysis to be carried out here, the spatial variation of the function $\hat{f}_{n}$ in the plane of the deformation is assumed as

$$
\hat{f}_{n}= \begin{cases}a & 0 \leqslant r \leqslant r_{o} \\ a \exp \left(\beta-\beta r^{2} / r_{o}^{2}\right) & r>r_{o} .\end{cases}
$$

Here $r$ is the radial distance measured from the centre of a particle, $r_{o}$ is its radius, and $\beta$ is a constant taken as less than unity which controls the decay rate of the exponential function in (16). The amplitude $a$ is chosen based on a unit cell model (see appendix A of Narasimhan (1992)), so that $\vec{f}_{n}$ is consistent with the radius of the particle $r_{o}$ and the interparticle spacing $d_{o}$.

On combining (3) to (7), along with plastic consistency and the evolution equations for the plastic internal variables $\left(\sigma_{m}\right.$ and $\left.f\right)$, the rate constitutive equation can be obtained in the form

$$
\sigma_{i j}^{*}=L_{i j k l}^{*} D_{k l} \text {, }
$$

where $L_{i j k l}$ is an elastic-plastic constitutive tensor. A numerical integration algorithm (see appendix B of Narasimhan (1992)) which ensures incremental objectivity is employed to update the stresses from (17) in the present work.

As noted above, complete loss of material stress-carrying capacity occurs when $f^{*}=f_{u}^{*}=1 / q_{1}$ (or equivalently when $f=f_{F}$ ) resulting in local material failure. This implies that the material completely separates at this point and a traction-free surface develops. This failure criterion was implemented in the numerical simulation by freezing the evolution of $f$ after it reaches a value close to $f_{F}$ (around $0.95 f_{F}$ ). The macroscopic material response is then elastic-perfectly plastic with a small pressuredependent yield stress. The condition $f=0.95 f_{F}$ was used instead of $f=f_{F}$ because as $f \rightarrow f_{F}$ the macroscopic equivalent stress $\sigma_{e} \rightarrow 0$, causing numerical difficulties.

\section{Numerical analyses}

Due to mode I symmetry, only one half of the notched three-point bend specimen was considered. It was modelled using (2-D) plane strain 4-noded bilinear elements as shown in figure 1a. The specimen has a total length of $160 \mathrm{~mm}$, initial crack length of $20 \mathrm{~mm}$, width of $40 \mathrm{~mm}$ and thickness of $20 \mathrm{~mm}$. The mesh used (see figure 1a) comprises a total of 556 nodes and 503 elements. In figure $1 \mathrm{~b}$, the details of the refined mesh which was used near the notch tip are displayed. The initial notch 


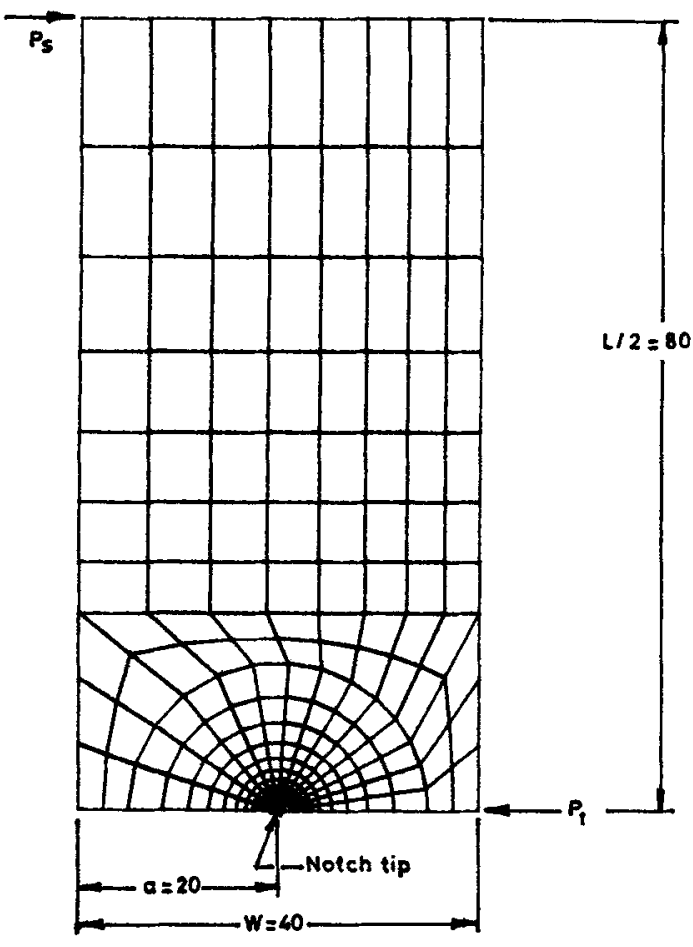

(a)

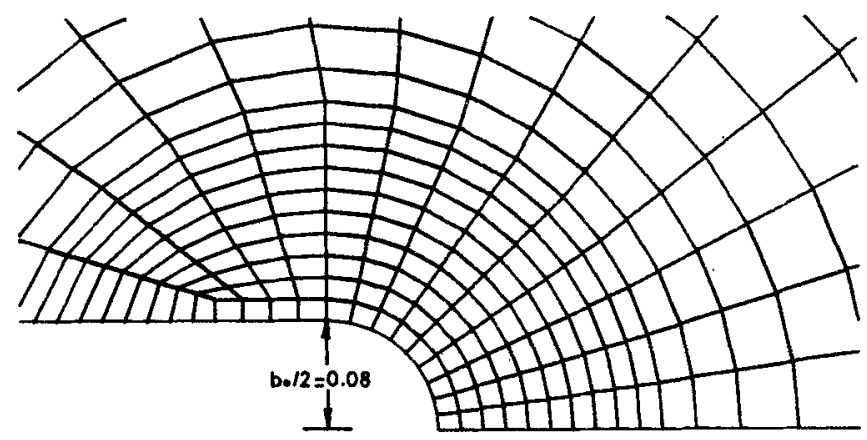

(b)

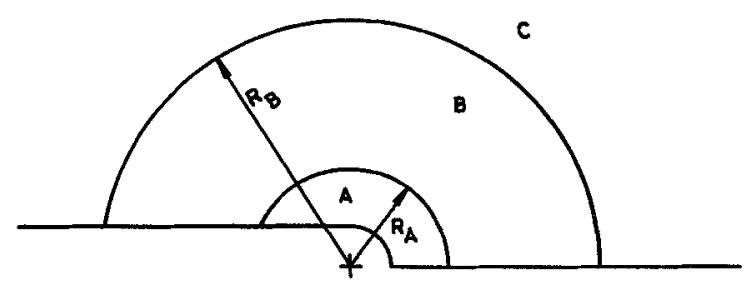

(c)

Figure 1. (a) Mesh used in the finite-element analysis. All dimensions are in mm. (b) Details of in-plane mesh near the notch tip. (c) Classification of region around the notch tip into $A, B$ and $C$ in dynamic analyses. 
diameter $b_{o}$ is $0 \cdot 16 \mathrm{~mm}$. The average dimension of the smallest element near the notch tip in the mesh shown in figure $1 \mathrm{~b}$ is approximately $0.014 \mathrm{~mm}$. Further, it should be observed from figure $1 \mathrm{~b}$ that the mesh near the notch tip is well refined and is hence expected to resolve the near-tip fields quite accurately. The finite-element procedure used in this work is based on an updated Lagrangian formulation that accounts for finite deformations and rotations (Narasimhan 1992).

The response of the matrix material in uniaxial tension (at constant test temperature) is assumed to be characterized by the power hardening law given in (8) and (9a). The reference stress $\sigma_{o}$ and the strain-hardening exponent $N$, obtained by fitting the functional form (9a) to the experimental stress-strain curve corresponding to room temperature and quasistatic loading, were $1000 \mathrm{MPa}$ and 0.04 , respectively. These values of $\sigma_{o}$ and $N$ were used in the analysis of the static fracture experiment. On the other hand, three different analyses were performed to simulate the dynamic drop-weight loading experiment corresponding to an initial impact speed of $6 \mathrm{~m} / \mathrm{s}$. In all the three analyses, inertial effects and thermal straining caused by adiabatic heating (see (6)) inside the plastic zone were considered.

In the first analysis, referred to as dynamic analysis \# 1 in the sequel, the chosen values of $\sigma_{o}$ and $N$ were the same as in the static analysis. In other words, strain rate sensitivity of the material was ignored. But thermal softening due to adiabatic heating was taken into account. Thus, the thermal softening parameter $\gamma$ in (9b) was taken as $4 \times 10^{-4} /{ }^{\circ} \mathrm{C}$, so that the reduction in yield stress with temperature matched with experimentally obtained data for temperatures ranging from $30^{\circ} \mathrm{C}$ to $400^{\circ} \mathrm{C}$.

In dynamic analysis \# 2 , thermal softening caused by adiabatic heating was ignored $(\gamma=0$ in $(9 b))$, but rate sensitivity due to high strain rates experienced by the material near the notch tip (which was of the order of $10^{3}$ to $10^{4} \mathrm{~s}^{-1}$ in the present analysis) was considered in an approximate fashion. To this end, the region near the notch tip (in the undeformed configuration) was divided into three zones as depicted in figure $1 \mathrm{c}$. In region $\mathrm{A}$, where the strain rates are expected to be maximum, the reference stress $\sigma_{o}$ and the hardening exponent $N$ were assumed as $1300 \mathrm{MPa}$ and 0.2 respectively to account for the increased yield stress and strain hardening. The radius $R_{\mathrm{A}}$ of this region with respect to the centre of the notch was taken as $1.5 b_{o}$. In region $B$, the strain rates experienced by the material are expected to be less than that in region $\mathrm{A}$. Hence, $\sigma_{o}=1100 \mathrm{MPa}$ and $N=0.1$ were used. The radius $R_{\mathrm{B}}$ of this region was taken as $4 b_{o}$. Finally, the background material in region $C$ was assumed to have the same value of $\sigma_{o}$ and $N$ as in the static analysis, since strain rate effects are expected to be very small here. It should be mentioned that the above method accounts for rate sensitivity in an approximate manner. A more realistic modelling of rate effects requires the use of viscoplastic constitutive equations. In dynamic analysis \# 3, both strain rate sensitivity (in the manner described above) and thermal softening (with $\gamma=4 \times 10^{-4} /{ }^{\circ} \mathrm{C}$ ) were included.

Since detailed microstructural data for the steel considered in this work were not available, the constants in the void nucleation laws (13) to (16) were chosen based on data published in the literature for similar high-strength alloy steels like 4340 steel. It is well known that in these alloy steel systems the typical inclusions present are iron carbides, manganese sulphides, aluminium oxides, etc.

The value of $\varepsilon_{n}$ was taken as 0.13 which is representative of the nucleation strain for cementite particles of size less than $0.1 \mu \mathrm{m}$ (see Goods and Brown 1979). A distribution of cementite particles of this size scale can be observed in (a similar) AISI 
4340 steel from the extraction replica, figure 3, given by Cox and Low (1974). A rather large standard deviation of $s_{n}=0 \cdot 1$ was used in the strain-controlled void nucleation law (14) to allow for scatter in the size of the small cementite particles. The value of $f_{n}$ in (14) was taken as 0.04 which roughly corresponds to a ratio of particle size to interparticle spacing of the above small particles of around $1 / 5$.

The spatial variation of $\hat{f}_{n}$ was assumed to be in the form (16), which is motivated by the consideration of a rectangular array of large particles that initiate voids by an interface stress-controlled mechanism. These particles were assumed to be circularcylindrical in shape with a diameter of $8 \mu \mathrm{m}$ and with a centre-to-centre spacing of $40 \mu \mathrm{m}$ (see, for example, table IIl of Cox and Low (1974)). Also, the decay parameter $\beta$ in (16) was taken as $0 \cdot 2$. The amplitude $a$ in (16) was obtained from a unit cell model (see appendix A of Narasimhan (1992)) as $0 \cdot 166$. In order to illustrate the distribution of the large simulated particles around the notch tip which nucleate voids by a stress-controlled mechanism, the contours of $\hat{f}_{n}=0.06$ are shown in figure 2 . The contours do not appear to be uniformly spaced and also do not have the circular shape which is expected from (16), because of the discretization introduced by the finite elements. Also, it must be noted that the above contours are much larger than the actual size of the particles that are being simulated. The value of $\sigma_{N}$ in (15) was taken as $1800 \mathrm{MPa}$, which is close to the critical interface stress reported by Argon and Im (1975) for carbide inclusions in steels. Again, a large standard deviation of $\hat{s}_{n}=500 \mathrm{MPa}$ was used to allow for scatter in the above chosen value of $\sigma_{N}$. Finally, the constant $c$ in (15) was taken as 0.6 .

The density $\rho_{o}$, elastic modulus $E$ and the Poisson's ratio were taken as $7800 \mathrm{~kg} / \mathrm{m}^{3}$, $200 \mathrm{GPa}$ and 0.3 respectively. The value of thermal expansion coefficient $\alpha$ and that of $\rho_{o} c_{p}$ were assumed as $1.2 \times 10^{-5} /{ }^{\circ} \mathrm{C}$ and $3.5 \mathrm{~N} /\left(\mathrm{mm}^{2}{ }^{\circ} \mathrm{C}\right)$ respectively. Since the

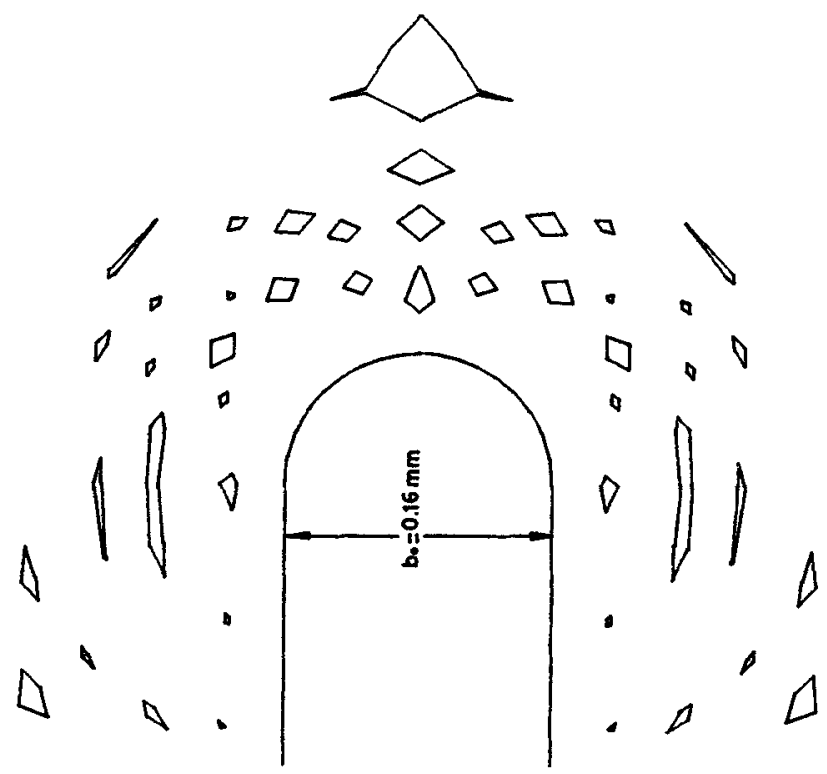

Figure 2. Contours of $\hat{f}_{n}=0.06$ illustrating the distribution of simulated inclusions surrounding the notch tip. 
plastic work density based on the undeformed material is used in this work (see (7)), the value of $\rho_{o} c_{p}$ was taken to pertain to the virgin (fully dense) matrix material.

\section{Results and discussion}

\subsection{Static loading}

A plane strain finite-element analysis of the static loading of the three-point bend specimen was performed using the mesh shown in figure 1 . The load point displacement was gradually incremented in small steps from the value which caused initial yielding in the element nearest to the notch root. The accuracy of the computation was continuously monitored by checking the magnitude of the out-of-balance forces.

In figure 3 , the variation of load with load point displacement obtained from the finite-element analysis is shown. In the experiments, crack initiation was observed at a load of $44,000 \mathrm{~N}$. The load point displacement corresponding to this load is found from figure 3 as around $0.6 \mathrm{~mm}$. It can be seen from figure 3 that the load increases almost linearly with load point displacement till the point at which crack initiation occurred in the experiments. In other words, the overall (or global) response of the specimen till crack initiation is almost linear. This is not surprising since the theoretical plane strain limit load (based on rigid perfectly-plastic idealization) for the specimen configuration and material used in this study is $72,800 \mathrm{~N}$. Thus, the crack initiation load is much lower than the limit load and plastic yielding is expected to be confined to a very small region near the notch tip.

In order to confirm the above assertion, the plastic zone (which is defined here as the contour of macroscopic equivalent stress $\sigma_{e}=\sigma_{0}$ ) is shown in figure 4 corresponding to a load of $44,000 \mathrm{~N}$. In this figure $x_{1}, x_{2}$ refer to Cartesian axes in the deformed

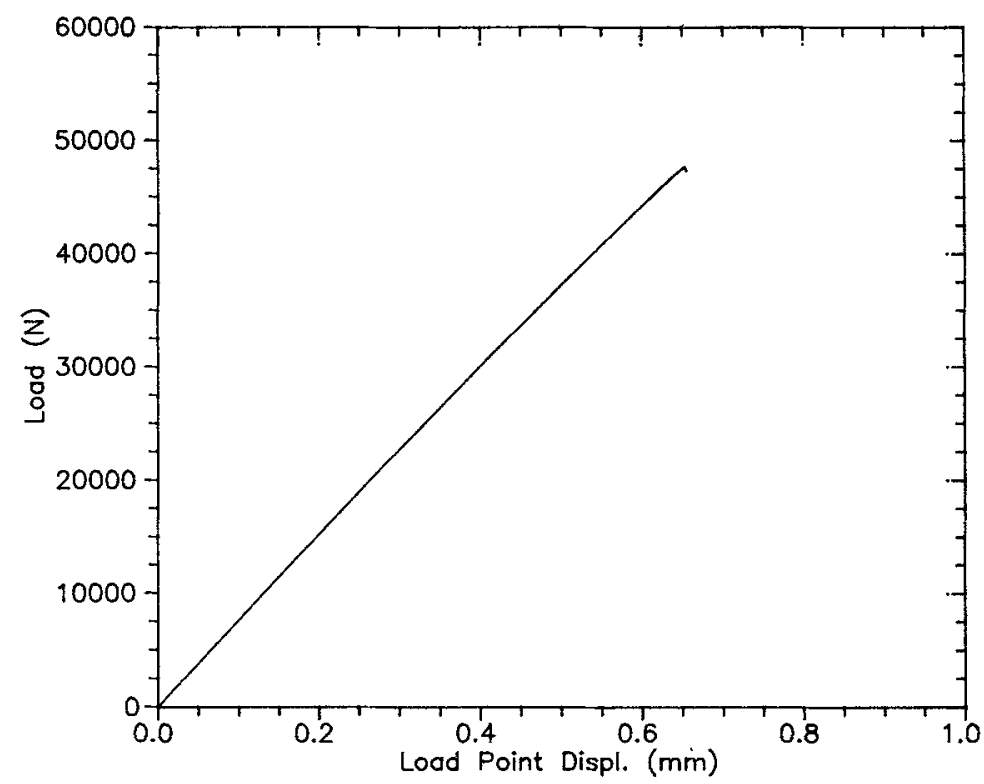

Figure 3. Load versus load point displacement in the static analysis. 


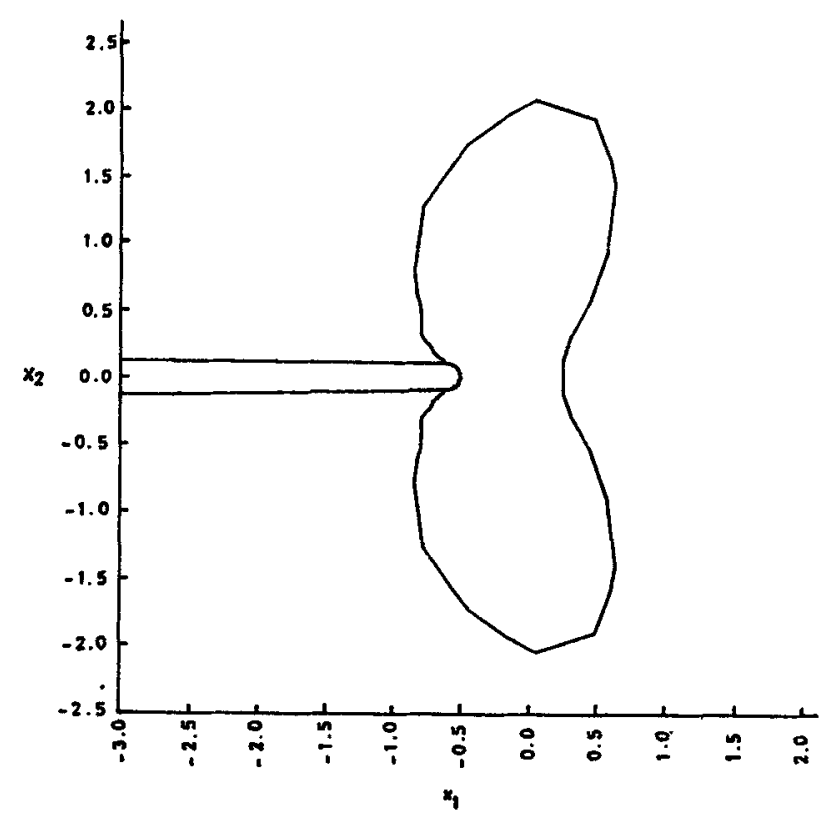

Figure 4. Plastic zone surrounding the notch tip in the deformed configuration at load of $44,000 \mathrm{~N}$ in the static analysis. The axes dimensions are in $\mathrm{mm}$.

configuration and the length dimensions are in $\mathrm{mm}$. It can be seen from figure 4 that the maximum plastic zone dimension is around $2.1 \mathrm{~mm}$ and occurs at an angle of about $70^{\circ}$ with respect to the notch tip. The maximum plastic zone size mentioned above compares well with the small-scale yielding results of Rice and Tracey (1973) based on a static, finite-element analysis for an elastic-perfectly plastic material obeying the $J_{2}$ flow theory. They have reported that the maximum plastic zone extent is equal to $0.15\left(K_{1} / \sigma_{o}\right)^{2}$, where $K_{1}$ is the mode I. stress intensity factor, and that it is attained at an angle of $70^{\circ}$ with respect to the crack tip. The value of the stress intensity factor $K_{\mathrm{I}}$ corresponding to a load of $44,000 \mathrm{~N}$ is computed on the basis of elastic estimates (Tada et al 1973) as $117 \mathrm{MPa} \sqrt{m}$. On using this value for $K_{1}$, the maximum plastic zone size as given by Rice and Tracey (1973) is found to be $2.05 \mathrm{~mm}$, which matches well with the present numerical results.

The finite strain version of the $J$ integral as defined by Eshelby (1970) was computed from the numerical solution using the domain integral formulation (Nakamura et al 1986). In figure 5 , the variation of $J$ with applied load is shown. The value of $J$ for the load range displayed in figure 5 compares well with that estimated on the basis of small-scale yielding. The small-scale yielding estimate of $\boldsymbol{J}$ for mode I plane strain is given by

$$
J=\left(1-v^{2}\right) K_{1}^{2} / E,
$$

where $K_{\mathrm{I}}$ is obtained from the load and specimen geometry (Tada et al 1973), $E$ is Young's modulus and $v$ is Poisson's ratio. For example, corresponding to the crack initiation load of $44,000 \mathrm{~N}$, the small-scale yielding estimate for $J$ is $62.3 \mathrm{kN} / \mathrm{m}$, which is very close to that obtained from the curve shown in figure 5. 


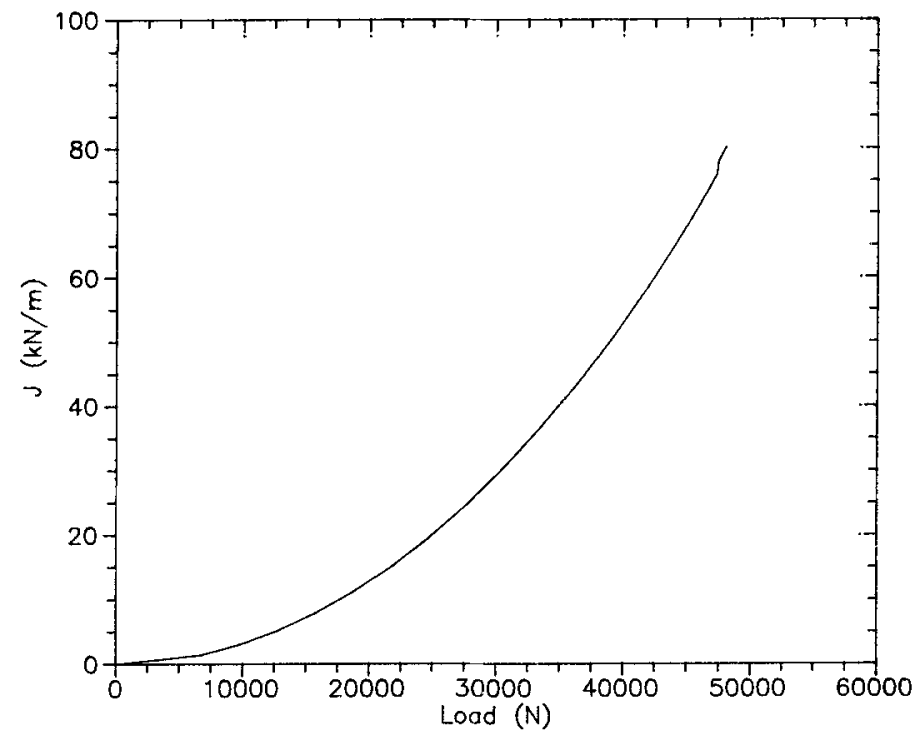

Figure 5. Variation of $J$ with load in the static analysis.

In figure 6 , the contours of hydrostatic stress $\sigma_{H}=\sigma_{k k} / 3$ are displayed around the deformed notch corresponding to a load of $44,000 \mathrm{~N}$. On examining the contours displayed in figure 6 , it is clear that the hydrostatic stress initially increases with distance ahead of the notch tip and reaches a maximum value at a point inside contour E. It decreases with distance beyond this point. The peak value of $\sigma_{H}$ is attained at a distance of $2 \cdot 5 b$, where $b$ is the current notch diameter in figure 6 , and is around $2 \cdot 3 \sigma_{o}$. This is in general agreement with analytical results (Rice and Rosengren 1968; Rice and Johnson 1970) for low-hardening materials. Further, it should be mentioned that the large value of the hydrostatic stress in the region ahead of the deformed notch will promote the nucleation and growth of microvoids. In addition to the above, it was found that the notch tip opening $\left(b-b_{0}\right)$ obtained from the present numerical results matched well with that estimated from the asymptotic, analytical HRR solution (Rice and Rosengren 1968) which is around $0.67 \mathrm{~J} / \sigma_{0}$. Thus, from the above discussion, it is clear that both global quantities such as the $J$ integral and the plastic zone size as well as local quantities such as the notch tip opening and the near-tip hydrostatic stress have been accurately computed by the present numerical solution.

The contours of matrix plastic strain $\varepsilon_{m}^{p}$ near the blunted notch surface (in the deformed configuration) corresponding to a load of $44,000 \mathrm{~N}$ are shown in figure 7 . The length scale in this figure is set by the current notch diameter $b$ which is equal to $0.205 \mathrm{~mm}\left(1 \cdot 28 b_{o}\right)$ at this stage. It can be seen from figure 7 that there is an intense concentration of matrix plastic strain at the vicinity of the notch tip. The value of $\varepsilon_{m}^{p}$ at the notch root in figure 7 is about 0.4 . It decreases sharply with distance ahead of the notch tip and reduces to a value of 0.04 (see contour A) at a distance of $1.5 b$ ahead of the tip.

The contours of void volume fraction near the blunted notch surface (in the deformed configuration) are presented in figure 8 at the stage when the load is $44,000 \mathrm{~N}$. As in figure 7, the length scale in this figure is set by the current notch diameter $b$. It can be observed from this figure that the void volume fraction is significant within 


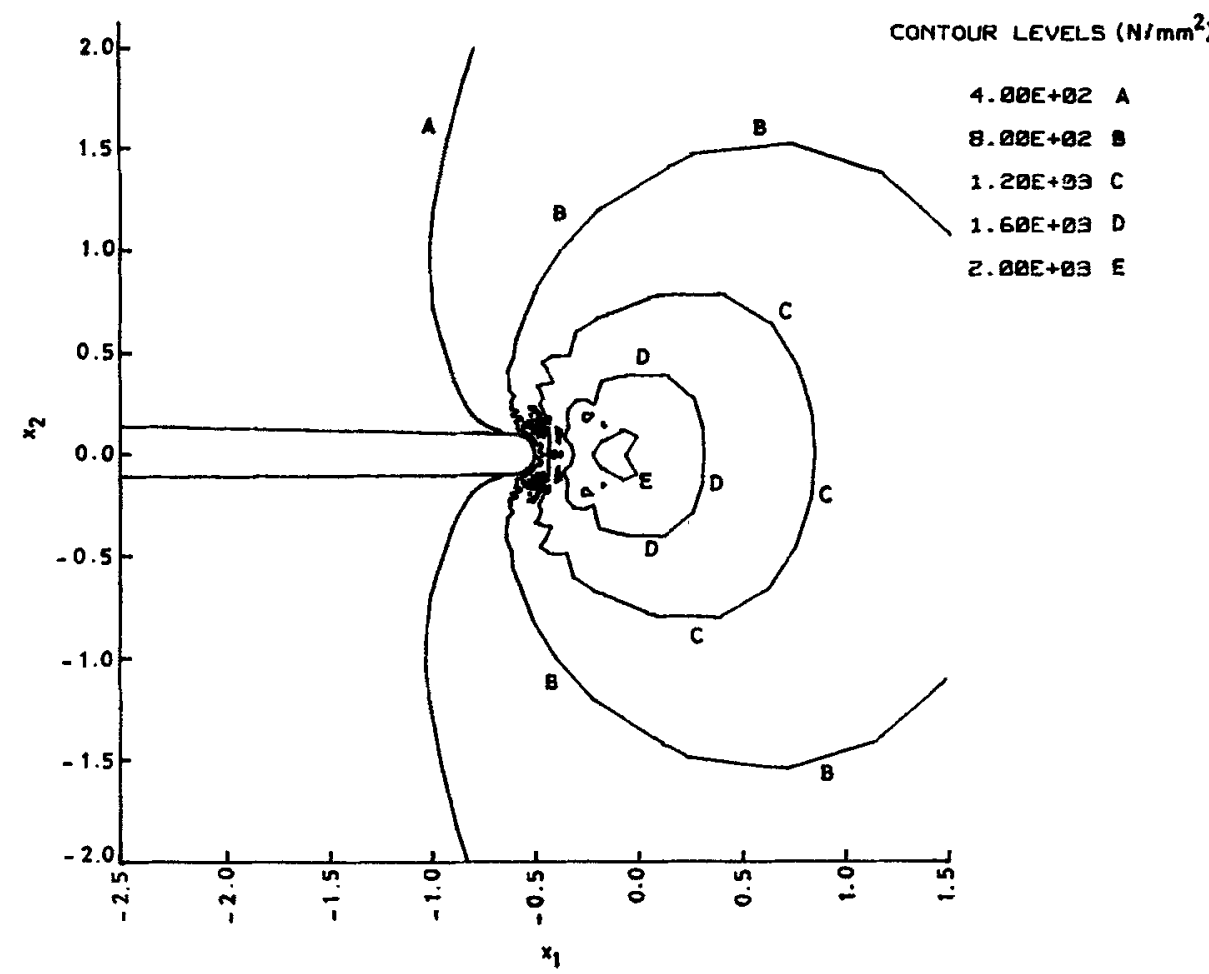

Figure 6. Contours of hydrostatic stress around the deformed notch obtained from the static analysis at load of $44,000 \mathrm{~N}$. The axes dimensions are in $\mathrm{mm}$.

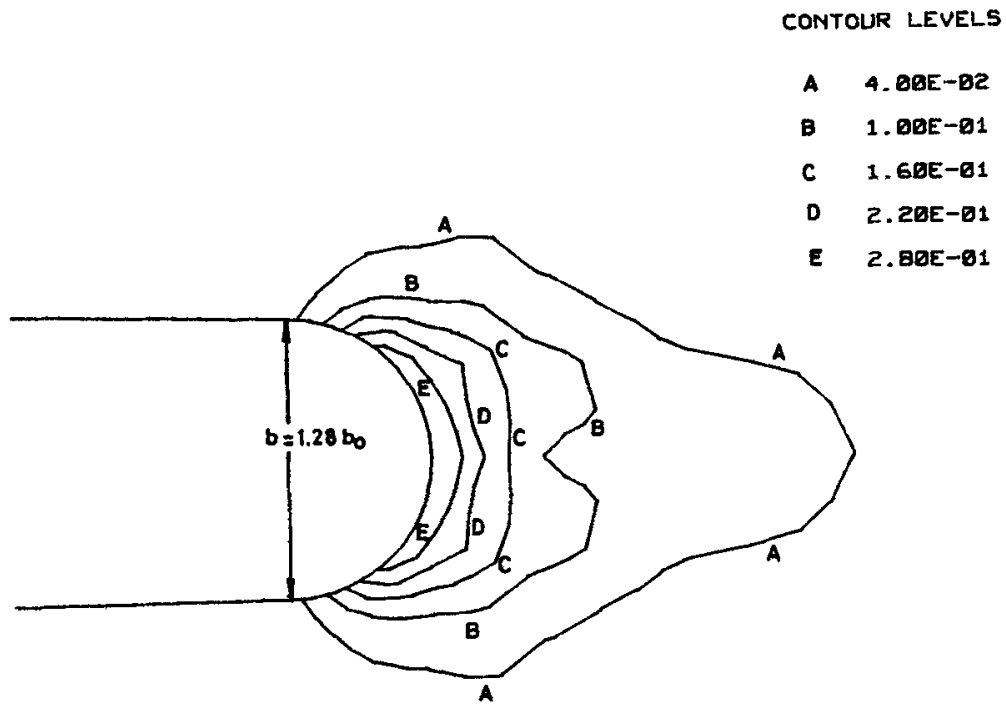

Figure 7. Contours of matrix plastic strain around the deformed notch at load of $44,000 \mathrm{~N}$. 


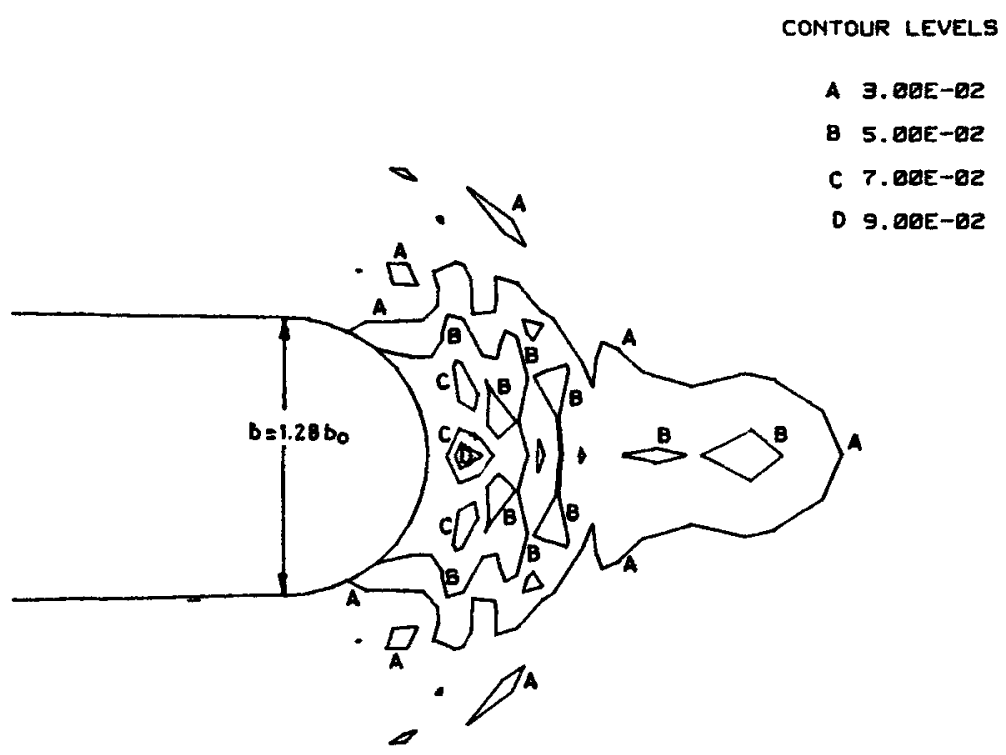

Figure 8. Contours of void volume fraction around the deformed notch at load of $44,000 \mathrm{~N}$.

the region enclosed by contour A which extends to a distance of about $2 b$ ahead of the notch tip. The contours corresponding to larger levels of $f$ appear as islands (see figure 8) which have formed around the sites of the simulated inclusions in this region. The large hydrostatic stress field in this region (which is enclosed between the notch surface and contour A) has led to nucleation of voids at the sites of the simulated inclusions. It should be recalled from $\S 2$ that void initiation at the large simulated inclusions occurs by a stress-controlled mechanism. This is followed by the growth of the nucleated voids around the simulated inclusions. The void growth rate is influenced both by large matrix plastic strain and hydrostatic stress. In particular, it should be noted that the void volume fraction is very large at the location of the first simulated inclusion ahead of the notch tip in figure 8. The maximum value of $f$ occurs within contour $\mathrm{D}$ in figure 8 and is around 0.13 which is close to the preset limit $f_{c}$ (see $\$ 2$ ) for the onset of microvoid coalescence.

Thus, the results from the numerical simulation carried out in this work demonstrate that corresponding to the load at which crack initiation was observed in the experiments, considerable amount of microvoid damage has accumulated in the region surrounding the notch tip. This is highlighted by the porosity distribution which indicates that microvoids have nucleated and grown around the simulated inclusions in this region. On further continuing the analysis, it was found that complete material failure due to microvoid coalescence occurred at some locations within the region enclosed by contour $A$ in figure 8 when $J$ attained a value of around $75 \mathrm{kN} / \mathrm{m}$.

\subsection{Dynamic loading}

The experimentally measured tup load corresponding to an impact speed of $6 \mathrm{~m} / \mathrm{s}$, which is plotted as a function of time in figure 9, was used as traction boundary condition in the dynamic analyses. A time step size of $1.8 \times 10^{-9} \mathrm{sec}$ was employed 


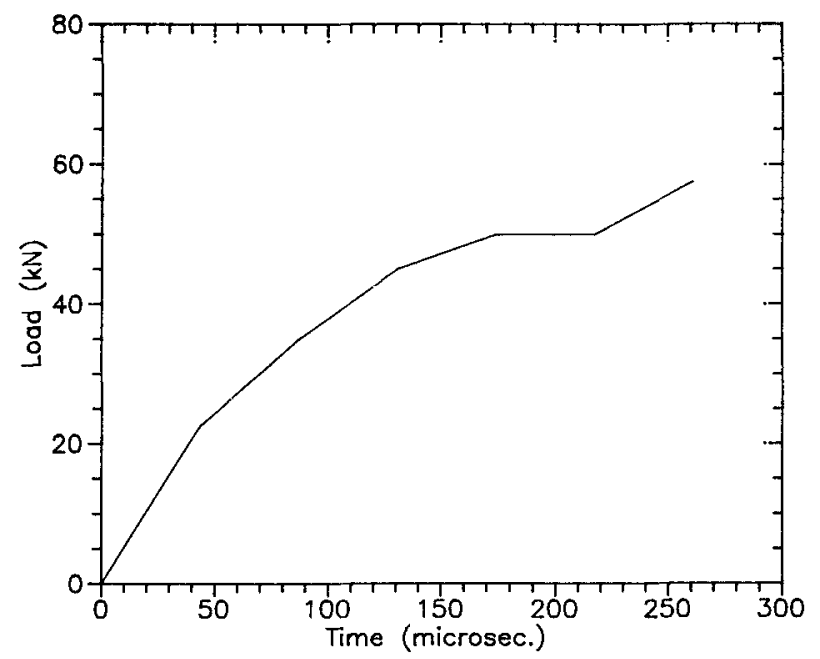

Figure'9. Experimentally obtained tup load versus time corresponding to impact speed of $6 \mathrm{~m} / \mathrm{s}$ which was used in the dynamic analyses.

in order to ensure that the numerical solution remains stable. Also, the stability of the numerical solution was continuously monitored by checking the global energy balance as suggested by Belytshko (1983) for nonlinear problems.

As mentioned in $\$ 3$, three different analyses were carried out with the view of assessing the individual and also the combined effects of thermal softening and material strain rate sensitivity, in addition to inertial effects. The results obtained from the three dynamic analyses regarding the time variation of global parameters such as the load point displacement, load point velocity, specimen energies and the dynamic $J$ integral were almost identical. Hence, these results computed from dynamic analysis \# 3 (see $\S 3$ ) alone will be presented below.

The dynamic finite-element analyses were supplemented by an elementary computation based on the mass-spring model as suggested by Williams (1987). For this analysis, the test specimen was idealized by a single degree of freedom oscillator (SDOF) with a linear spring as in the work of Zehnder et al (1990). The spring stiffness $k$ was chosen to be the same as the (elastic) stiffness of the test specimen which is equal to $7.92 \times 10^{7} \mathrm{~N} / \mathrm{m}$. The effective mass, $m$, of the model is chosen so that the kinetic energy of the model and the specimen are equal when the model and the specimen have the same load point velocities (see Williams (1987) for details). This effective mass $m$ of the model was estimated as $0.5 \mathrm{~kg}$. The analysis was carried out by applying the experimentally measured tup load (see figure 9) to the SDOF and by integrating the equation of motion numerically. The load point displacement and load point velocity obtained from the above computation are employed to calculate the specimen internal energy, kinetic energy and the spring reaction force. The spring reaction force is used along with the expression given by Tada et al (1973) corresponding to the test specimen geometry to compute the dynamic stress intensity factor, and hence the dynamic $J$ integral.

The variation of the load point displacement and load point velocity (load point displacement rate) with time are shown in figures 10 and 11 , respectively. The results obtained from the dynamic finite-element analysis \# 3 (see $\$ 3$ ) and from the analysis 


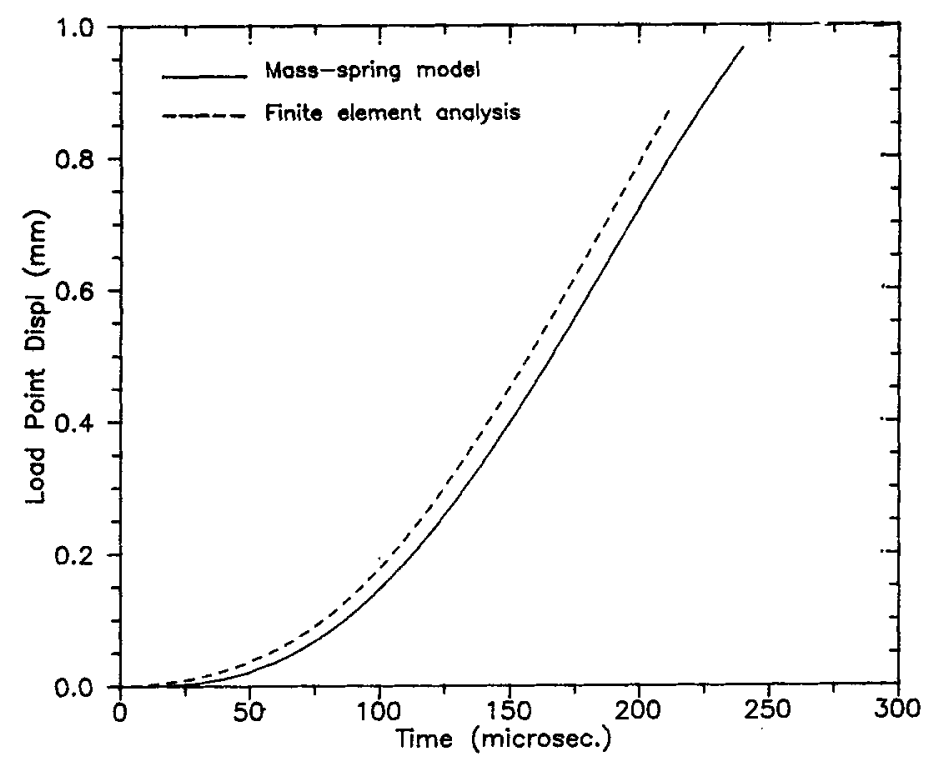

Figure 10. Load point displacement versus time obtained from dynamic analysis \# 3 and the mass-spring model.

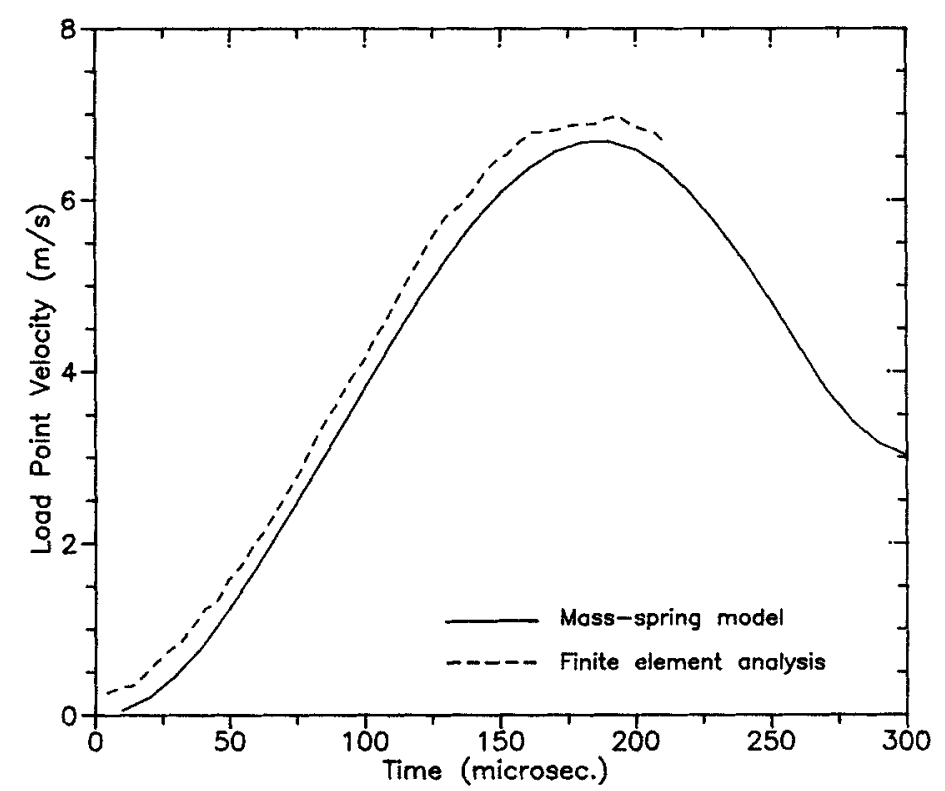

Figure 11. Load point velocity versus time obtained from dynamic analysis \#3 and the mass-spring model.

based on the mass-spring model are presented in these figures. It can be seen from these figures that the load point displacement and velocity computed from the mass-spring model compare quite well with the more accurate finite-element results, although they are about 5 to $10 \%$ lower than the latter. It can be observed from 


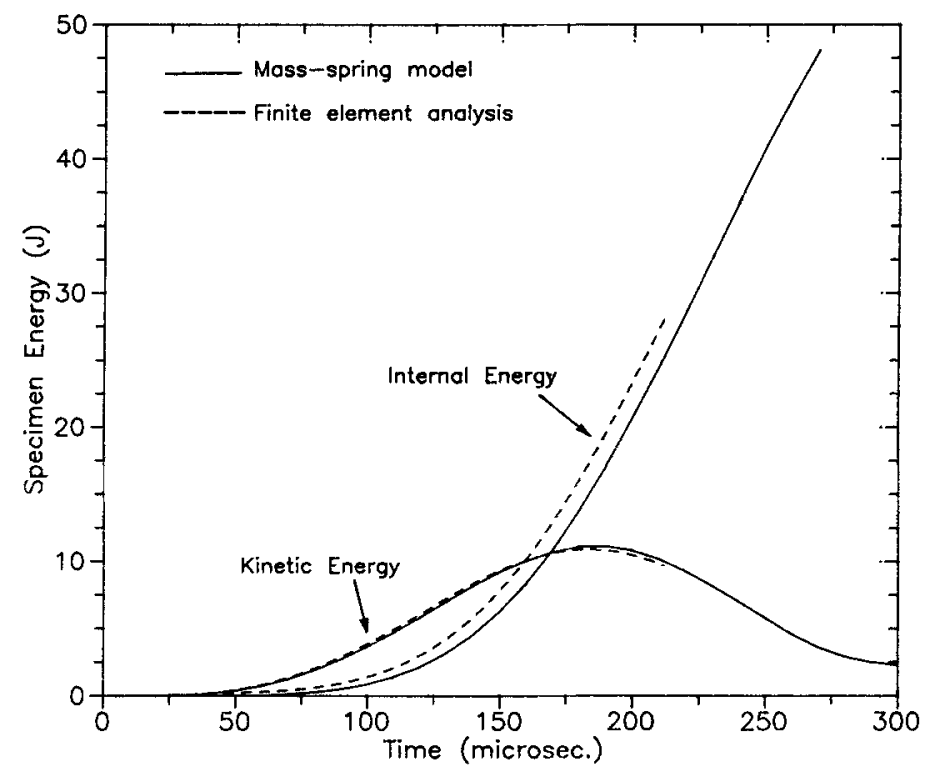

Figure 12. Specimen energy (in joules) versus time obtained from dynamic analysis \# 3 and the mass-spring model.

figure 10 that the load point displacement increases monotonically with time and attains a value of around $0.8 \mathrm{~mm}$ at $200 \mu \mathrm{s}$. On the other hand, figure 11 shows that the load point velocity increases to a peak value of about $6.9 \mathrm{~m} / \mathrm{s}$ at around $185 \mu \mathrm{s}$ and thereafter decreases. In fact, the mass-spring model analysis which was continued beyond $300 \mu$ s indicated that the load point velocity decreases to a minimum and again starts increasing.

In figure 12, the time variation of the internal energy (deformation energy) and kinetic energy of the specimen, computed from the dynamic finite-element analysis \# 3 and the mass-spring model, are displayed. As in figures 10 and 11, it can be seen from figure 12 that the results obtained from the simple mass-spring model compare reasonably well with the more accurate finite-element analysis. It may be observed that the kinetic energy of the specimen is higher than the internal energy during the early phase of loading. For example, at a time of $125 \mu \mathrm{s}$, the kinetic energy and internal energy are 6.8 joules and 3.7 joules respectively. The specimen internal and kinetic energies become equal at around $160 \mu \mathrm{s}$. The internal energy continues to increase beyond this stage and attains a value of about 24 joules at $200 \mu \mathrm{s}$. The kinetic energy, on the other hand, reaches a peak value of 11 joules at $185 \mu \mathrm{s}$ and thereafter decreases, which conforms to the time variation of the load point velocity (see figure 11).

In figure 13 , the time variation of the dynamic $J$ integral, $J_{d}$, which was computed using the domain integral representation (Nakamura et al 1986) from the finite-element results (corresponding to analysis \# 3 ), is shown along with that obtained from the mass-spring model analysis. As before, the time record computed from the mass-spring model matches well with the finite-element results. Thus, from figures 10 to 13 , it can be concluded that the time variation of the global parameters depicted in these figures may be determined quite accurately using the mass-spring model. It should be noted in this connection that stress-wave effects (except in the initial phase of loading) are 


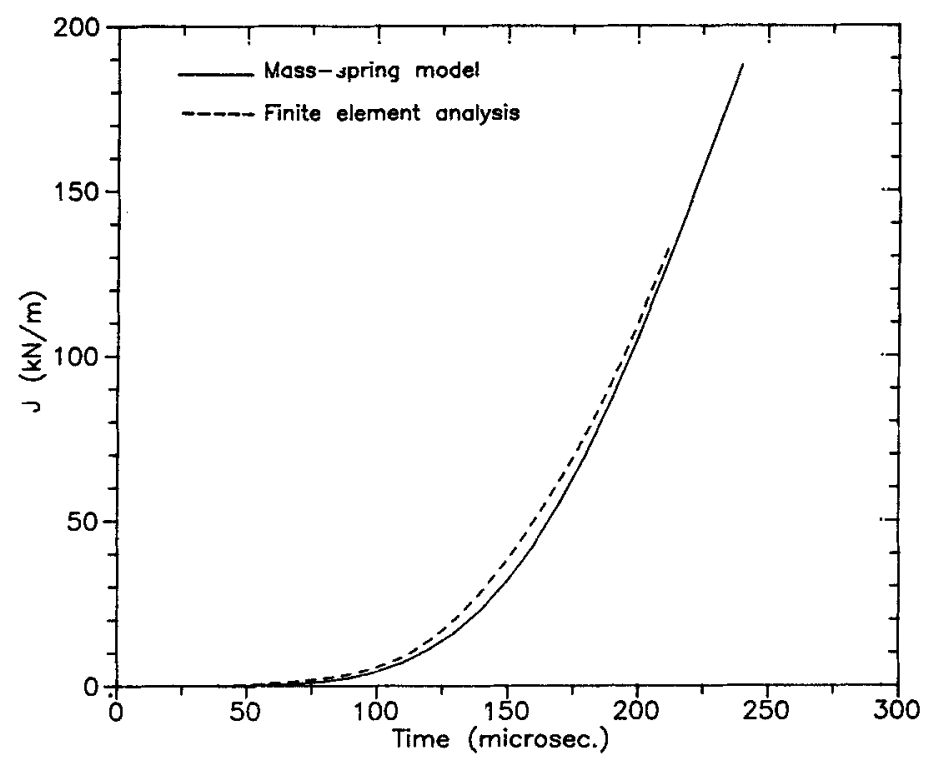

Figure 13. Variation of the dynamic $J$ integral with time obtained from dynamic analysis \# 3 and the mass-spring model.

not very dominant in the experiment analysed in the present work and also plastic yielding is contained in a small region near the crack tip (see, for example, figure 4). A similar observation was made by Zehnder et al (1990) after comparing the results for $J_{d}$, obtained using the mass-spring model analysis of a similar three-point bend test specimen geometry with both finite-element results and experimental results based on the shadow optical method of caustics. This makes the mass-spring model really attractive for analysing low-velocity impact experiments of fracture specimens since it is very simple and also takes very little computer time compared to an elaborate dynamic finite-element analysis.

It can be observed from figure 13 that $J_{d}$ increases monotonically with time and attains a value of $110 \mathrm{kN} / \mathrm{m}$ at $200 \mu \mathrm{s}$. An average value of $j$ over the time range from 0 to $200 \mu \mathrm{s}$ is found to be $5.5 \times 10^{5} \mathrm{kN} / \mathrm{m} \mathrm{s}$. As noted by Jha and Narasimhan (1992) and Narasimhan (1992) a rapid increase in $J_{d}$ with time allows for a $J$-controlled field (Rice and Rosengren 1968) to stabilize near the notch tip. It also results in rapid evolution of micromechanical quantities and to the accumulation of microvoid damage near the notch tip.

In figure 14, the contours of void volume fraction, obtained from dynamic analysis \# 3 at a time of $210 \mu \mathrm{s}$, are shown in the deformed configuration surrounding the notch tip. The value of $J_{d}$ is equal to $130 \mathrm{kN} / \mathrm{m}$ at this time and the current notch diameter $b=0.228 \mathrm{~mm}\left(1.42 b_{o}\right)$. As in the static analysis (see figure 8), it can be observed from figure 14 that the contours of $f$ encircle the sites of the simulated inclusions around the notch tip. It is important to note that at the stage shown in figure 14 , the element located at the site of the first simulated inclusion ahead of the notch tip (see contour $\mathrm{D}$ in figure 14) has already experienced complete material failure. In fact, the void volume fraction in the entire ligament connecting the first simulated inclusion with the notch tip (which is enveloped by contour B in figure 14) is very high. 


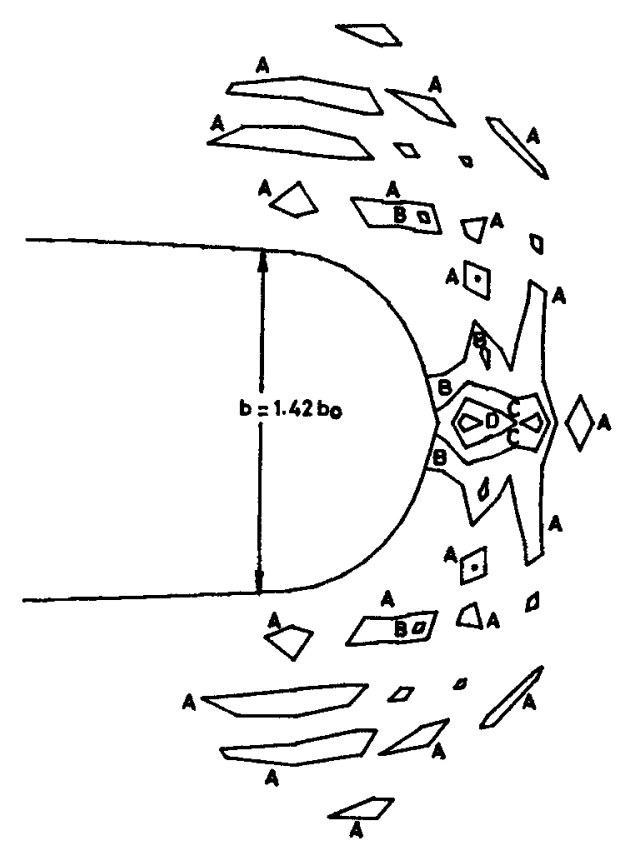

Figure 14. Contours of void volume fraction around the deformed notch at $t=210 \mu \mathrm{s}$ based on dynamic analysis \# 3. (A) $6 \cdot 00 \mathrm{E}+02$, (B) $1 \cdot 1 \mathrm{E}+01$, (C) $1 \cdot 6 \mathrm{E}+01$ and (D) $2 \cdot 1 \mathrm{E}+01$.

In order to understand the magnitude of temperature rise experienced by the material near the notch tip due to plastic dissipation, the contours of accumulated plastic work density $W^{p}$ per unit undeformed volume are shown in figure 15 . These contours are based on the results of dynamic analysis \# 3 and correspond to $t=210 \mu \mathrm{s}$. As mentioned in $\S 2$, adiabatic conditions are assumed to prevail near the notch tip under dynamic loading, so that the temperature rise $\Delta T \approx \chi W^{p} / \rho_{o} c_{p}$ (see (7)), where $\chi=0.9$. It can be seen from figure 15 that the plastic work density is intensely concentrated in a small region near the notch tip. The plastic work density pertaining to contour $D$ in figure 15 is $1150 \mathrm{~N} / \mathrm{mm}^{2}$. This corresponds to an adiabatic temperature rise of $\Delta T \approx 296^{\circ} \mathrm{C}$. The plastic work density decreases to $100 \mathrm{~N} / \mathrm{mm}^{2}$ (see contour A) which corresponds to $\Delta T \approx 26^{\circ} \mathrm{C}$ at a distance of $0.5 b\left(0.71 b_{o}\right)$ ahead of the notch tip.

The evolution of void volume fraction $f$ with the $J$ integral in the element located at the notch root is shown in figure 16 . The results obtained from the static analysis and from the three dynamic analyses which were undertaken are presented in this figure. The corresponding curves, depicting the evolution of $f$ with $J$ in the element located at the position of the first simulated inclusion (see figure 2) ahead of the notch tip (at a distance of $X_{1}=0.04 \mathrm{~mm}$ in the undeformed configuration), are displayed in figure 17. These figures help in understanding the effects of strain rate sensitivity, adiabatic heating and material inertia on the accumulation of microvoid damage near the notch tip.

On examining figure 16 , it can be seen that the notch root element exhibits the fastest evolution of void volume fraction with $J$ under static loading. The variation of $f$ with $J$ in dynamic analysis \# 1 is slower than in the static analysis. This is attributed 


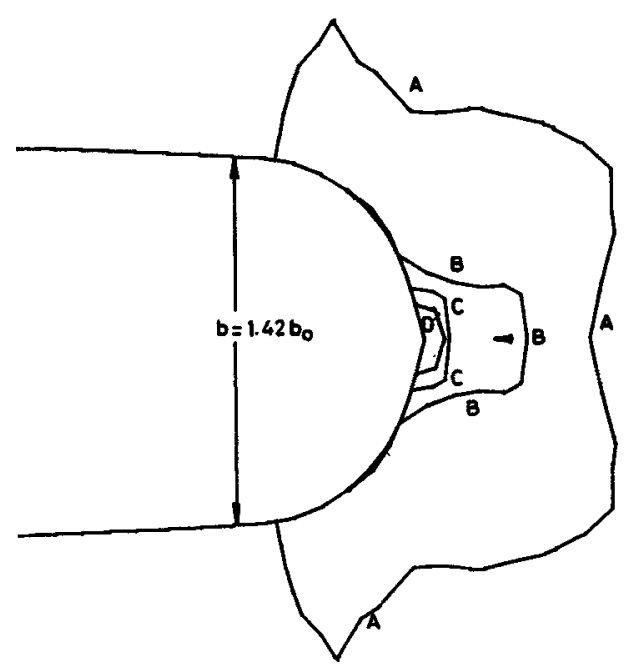

Figure 15. Contours of plastic work density (per unit undeformed volume) near the notch tip at $t=210 \mu \mathrm{s}$ from dynamic analysis \#3. Contour levels $\left(\mathrm{N} / \mathrm{mm}^{2}\right)$ : (A) $1.00 \mathrm{E}+02$, (B) $4 \cdot 50 \mathrm{E}+02$, (C) $8 \cdot 00 \mathrm{E}+02$ and (D) $1 \cdot 15 \mathrm{E}+03$.

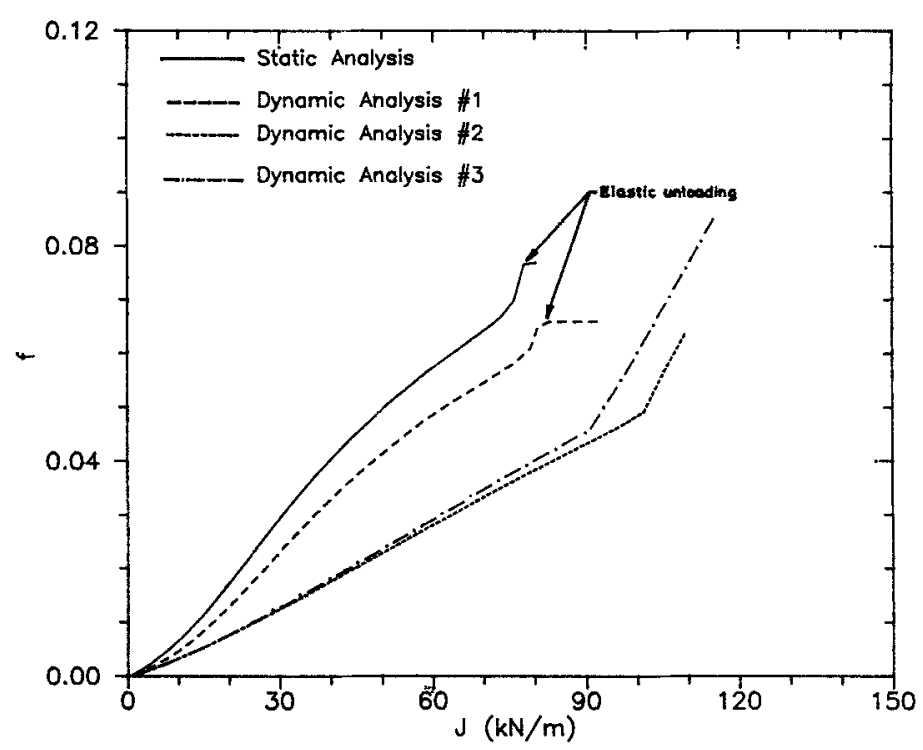

Figure 16. Evolution of void volume fraction in the element located at the notch root with $J$ in the static analysis and the three dynamic analyses.

purely to the effect of inertia of the matrix material in reducing the void growth rate, which is in fact opposed by thermal softening of the matrix material (caused by adiabatic heating) in dynamic analysis \# 1 . The notch root element experienced elastic unloading in the above two analyses at the stages indicated in figure 16 due to complete material failure in an adjacent element.

It may be observed from figure 16 that the slowest evolution of $f$ with $J$ at the notch root occurs in dynamic analysis \#2 in which the individual effects of strain rate 


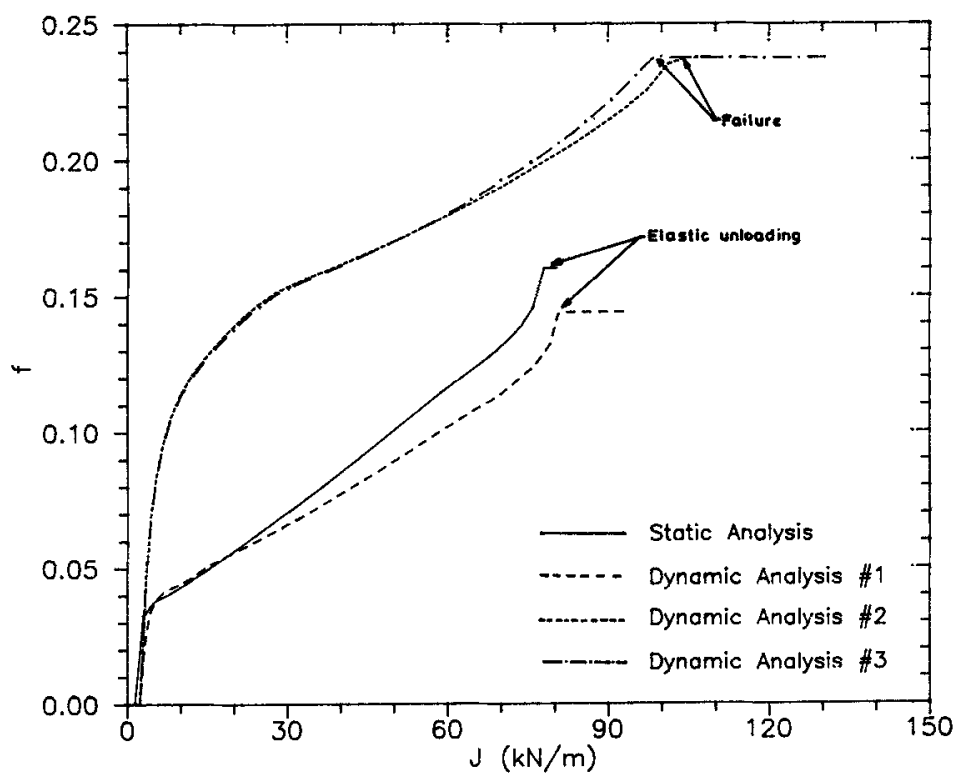

Figure 17. Evolution of void volume fraction in the element located at the site of the first simulated inclusion ahead of the notch tip with $J$.

sensitivity (in addition to inertial effects) were taken into account. The primary reason for this is the reduced void growth rate caused by the enhanced strain hardening of the matrix material which was incorporated in this analysis (see $\S 3$ ). The above assertion is corroborated by the work of Thomason (1990) who has integrated the Rice and Tracey (1969) equations describing the growth rate of a spherical void in a rigid plastic material. His results show (see, in particular, figures $2 \cdot 11$ to $2 \cdot 13$ in Thomason (1990)) that the presence of strain hardening reduces the void growth rates and that this effect is more dramatic at higher hydrostatic stress levels. Finally, when the opposing effects of thermal softening and strain rate sensitivity were simultaneously included in dynamic analysis \# 3 , it is found that the accumulation of void volume fraction with $J$ at the notch root is only slightly faster than in analysis \#2, but is still significantly slower than in analysis \# 1 (see figure 16).

In order to understand the quantitative effect of the individual factors discussed above, the value of $J$ required to attain a certain level of $f$ at the notch root in all the analyses carried out in this work can be compared. For example, taking a value of $f=0.06$, it can be deduced from figure 16 that it is attained at the notch root when $J=64 \mathrm{kN} / \mathrm{m}$ in the static analysis, whereas it is reached at $J=78 \mathrm{kN} / \mathrm{m}$, $107 \mathrm{kN} / \mathrm{m}$ and $100 \mathrm{kN} / \mathrm{m}$ in the three dynamic analyses respectively. Thus, compared to the static analysis, the value of $J$ (in order to reach a value of $f=0.06$ at the notch root) is $22 \%, 67 \%$, and $56 \%$ higher in the three dynamic analyses.

The curves presented in figure 17 which depict the accumulation of $f$ in the element located at the site of the first simulated inclusion ahead of the notch tip are somewhat different from those presented in figure 16. It can be seen from figure 17 that during the early phase of loading (as characterized by small values of $J$ ), a rapid evolution of $f$ occurs in dynamic analyses \#2 and \#3. This is because of the elevation in the matrix flow stress caused by strain rate sensitivity which enables the early attainment 
of the critical interface stress that is required to nucleate a void at the first simulated inclusion ahead of the notch tip. It should be noted that, during this stage, very little thermal softening occurs because the temperature rise has not yet reached a significant level and, hence, the curves corresponding to dynamic analyses \# 2 and \# 3 in figure 17 are almost identical.

Once the void nucleation phase at the first simulated inclusion ahead of the notch tip is completed, further evolution of $f$ takes place by void growth. In this phase, as noted earlier, the increased strain hardening considered in dynamic analyses \# 2 and \# 3 tends to slow down the accumulation of $f$ with respect to $J$. This can be clearly seen by comparing the slope of the $f$ vs $J$ curves (for $J$ greater than, say, $30 \mathrm{kN} / \mathrm{m}$ ) corresponding to dynamic analysis \# 2 and \# 3 with that for analysis \# 1 and for the static case. Since the temperature rise due to adiabatic heating becomes significant only at later stages of loading, the curves pertaining to dynamic analyses \#2 and \# 3 begin to differ only for $J$ greater than about $70 \mathrm{kN} / \mathrm{m}$. As expected, the curve for analysis \# 3 goes above that for analysis \# 2 because thermal softening is included in the former, which works against strain rate sensitivity and, thus, opposes the retardation of void growth rate.

As in the case of figure 16, it is to be noted that elastic unloading has occurred in the element located at the first inclusion ahead of the notch tip in the static analysis and in dynamic analysis \# 1, at the stages indicated in figure 17 . This has been caused by complete material failure in a neighbouring element when $J=76 \mathrm{kN} / \mathrm{m}$ and $81 \mathrm{kN} / \mathrm{m}$ for the static analysis and dynamic analysis \# 1 respectively. By contrast, in dynamic analyses \#2 and \#3, the void volume fraction in the element under consideration in figure 17 increases further and complete failure occurs when $J=105 \mathrm{kN} / \mathrm{m}$ and $98 \mathrm{kN} / \mathrm{m}$ respectively.

To summarize, the results presented in figures 16 and 17 indicate that material inertia, by itself, for the rate of loading considered in this work, marginally decelerates the void growth rate near the notch tip. The elevation in matrix flow stress due to strain rate sensitivity under dynamic loading promotes early void nucleation at the site of the first inclusion ahead of the notch tip. But it does not have an appreciable effect on void nucleation at the notch root which happens by a strain-controlled mechanism. The factor that has the greatest influence in slowing down the accumulation of microvoid damage near the notch tip under dynamic loading is increased strain hardening caused by material strain rate sensitivity. This would be reflected by a significant enhancement of the dynamic fracture toughness as characterized by the value of $J$ at crack initiation. The enhancement in the critical value of $J$ required to produce ductile failure near the notch tip will be of the order of $50 \%$ over the static fracture toughness in the present work. On the other hand, thermal softening caused by adiabatic heating has the opposite effect of accelerating the void growth rate near the notch tip. Thus, its individual effect would be to diminish the dynamic (ductile) fracture toughness, although in the present computations, its role is found to be much less significant than that of strain rate sensitivity.

\section{Conclusions}

In this work, static and dynamic fracture experiments using a three-point bend specimen of a high-strength low-alloy steel (SPADE), conducted at DMRL, Hyderabad, have 
been modelled by plane strain finite-element simulations. The main conclusions of the analyses are summarized below.

(i) The maximum plastic zone size at the (experimental) crack initiation load of $44,000 \mathrm{~N}$ in the static analysis is found to be around $2.1 \mathrm{~mm}$. The numerical results indicate that small-scale yielding conditions prevail at crack initiation.

(ii) The hydrostatic stress attains a peak value of $2 \cdot 3 \sigma_{o}$ at a distance of $2 \cdot 5 b$ (current notch diameters) ahead of the notch tip.

(iii) An examination of porosity distribution corresponding to the crack initiation load in the static analysis shows that considerable amount of ductile damage has accumulated near the notch tip. The contours of void volume fraction encircle the simulated inclusions near the notch tip. In the numerical simulation of static fracture initiation, complete material failure was detected in the vicinity of the notch tip when $J=75 \mathrm{kN} / \mathrm{m}$.

(iv) The time variation of global parameters such as load point displacement and velocity, specimen energies and dynamic $J$ integral, computed using the simple mass-spring model, compare well with more accurate and elaborate finite-element analyses. The average value of $\dot{J}$ in the time range from 0 to $200 \mu \mathrm{s}$ is found to be $5.5 \times 10^{5} \mathrm{kN} / \mathrm{ms}$.

(v) A study of the evolution of void volume fraction near the notch tip shows that increased strain hardening caused by strain rate sensitivity, which becomes important under dynamic loading, has the greatest effect in retarding the void growth rates near the notch tip. This could manifest itself in the present context in an enhancement of the dynamic fracture toughness (as characterized by the critical value of $J$ for the onset of ductile failure near the notch tip) by about $50 \%$ as compared to the static case.

(vi) By contrast, thermal softening caused by adiabatic heating (due to plastic dissipation) has the opposite effect of enhancing the void growth rate. However, quantitatively, it appears to be less significant than strain rate sensitivity in the present analysis.

\section{Acknowledgements}

The first author wishes to gratefully acknowledge the Department of Science and Technology (Government of India) for financial support through sponsored Project No. III-5 (48)/89-ET. The work reported here forms part of this sponsored project. The first author also wishes to thank Prof. E S Dwarakadasa, Department of Metallurgy, Indian Institute of Science, Bangalore, for helpful discussions and suggestions regarding this work.

\section{References}

Andersson H 1977 J. Mech. Phys. Solids 25217

Argon A S, Im J and Safogulu R 1975 Metall. Trans. A6 825

Argon A S and Im J 1975 Metall. Trans. A6 839

Belytshko T 1983 in Computational methods for transient analysis (eds) T Belytshko and T J R Hughes (Amsterdam: Elsevier) p. 1 
Brown L M and Embury J D 1973 in Microstructure and design of alloys, Proc. of the third intl. conf. on the strength of metals and alloys (England: Cambridge) p. 164

Chu C C and Needleman A 1980 J. Engg. Mater. Technol. 102249

Cox T B and Low J R 1974 Metall. Trans. 51457

Eshelby J D 1970 in Inelastic behaviour of solids, (eds) M F Kanninen, W F Adler, A R Rosenfield and R I Jaffee (New York: McGraw Hill) p. 77

Goods S H and Brown L M 1979 Acta Metall. 271

Gurson A L 1977 J. Engg. Mater. Technol. 992

Hahn G T and Rosenfield A R 1975 Metall. Trans. A6 653

Jha M and Narasimhan R 1992 Int. J. Fract. 56209

McMeeking R M 1977 J. Mech. Phys. Solids 25357

Nakamura T, Shih C F and Freund L B 1986 Engg Fract. Mech. 25323

Narasimhan R 1992 Engg. Fract. Mech. (to appear)

Needleman A and Rice J R 1978 in Mechanics of sheet metal forming (eds) D P Koistinen and N M Wang (New York: Plenum Press) p. 237

Needleman A and Tvergaard V 1987 J. Mech. Phys. Solids 35151

Needleman A and Tvergaard V 1991a Int. J. Fract. 4941

Needleman A and Tvergaard V 1991b Engg. Fract. Mech. 38157

Priest A H 1976 in Proc. of int. conf. on dynamic fracture toughness, Welding Institute (England: Cambridge) p. 95

Rice J R and Johnson M A 1970 in Inelastic behaviour of solids (eds) M F Kanninen, W F Adler, A R Rosenfield and R I Jaffee (New York: McGraw Hill) p. 641

Rice J R and Rosengren G F 1968 J. Mech. Phys. Solids 161

Rice J R and Tracey D M 1969 J. Mech. Phys. Solids 17201

Rice J R and Tracey D M 1973 in Numerical methods in structural mechanics (eds) S J Fenves et al (New York: Academic Press) p. 585

Tada H, Paris P C and Irwin G R 1973 The stress analysis of cracks handbook (Hellertown, Pa: Del Research Corp.)

Taylor G I and Quinney M A 1934 Proc. R. Soc. Series A 143307

Thomason P F 1990 Ductile fracture of metals (Oxford: Pergamon Press)

Tvergaard V 1981 Int. J. Fract. 17389

Tvergaard V and Needleman A 1984 Acta Metall. 32157

Williams J G 1987 Int. J. Fract. 33209

Zehnder A T, Rosakis A J and Krishnaswamy S 1990 Int. J. Fract. 42209 